\title{
Quelle stratégie française dans le cadre H2020 ?
}

\author{
Jean-François Dhainaut ${ }^{1,2}$, Vincent Diebolt ${ }^{3}$, Brigitte Pouletty-Lefebure et les participants à la table ronde $N^{\circ} 6$ \\ de Giens XXX : Angela Baker ${ }^{5}$, François Bassompierre ${ }^{1}$, Thomas Borel ${ }^{6}$, David Braunstein ${ }^{7}$, Jacques Demotes ${ }^{8}$, \\ Bruno François ${ }^{9}$, Stéphane Huet ${ }^{10}$, Joëlle Micallef ${ }^{11}$, Christophe Misse ${ }^{1}$, Annamaria Molon ${ }^{12}$, Olivier Rascol ${ }^{13}$, \\ Sophie Ravoire ${ }^{14}$, Bertrand Schwartz ${ }^{15}$ et avec la contribution sous forme d'audition de Nathalie Donne ${ }^{16}$, \\ Guillaume Fusaï ${ }^{17}$, Philippe Pouletty ${ }^{18}$ et Eric Vicaut ${ }^{19+}$
}

1 DRCD, AP-HP, Hôpital Saint Louis, Paris, France

2 GIRCI Ile-de-France, Hôpital Saint Louis, Paris, France

3 F-CRIN, Toulouse, France

4 Laboratoire Sanofi France, Paris, France

5 Inserm Transfert, Paris, France

6 Laboratoire Boehringer Ingelheim, Paris, France

7 AP-HM, Marseille, France

8 ECRIN, Paris, France

9 CHU de Limoges, Limoges, France

10 Laboratoire Glaxosmithkline, Les Ulis, France

11 Pharmacologie Clinique et Pharmacovigilance, AP-HM, Marseille, France

12 CIC CPCET, Hôpital de la Timone, Marseille, France

13 CHU Purpan, Toulouse, France

14 SR Consulting, Paris, France

15 Agence Nationale de la Recherche, Paris, France

16 DBV Technologies, Bagneux, France

17 Ministère de l'Education Nationale, de l'Enseignement Supérieur et de la Recherche, Paris, France

18 Truffle Capital, Paris, France

19 Hôpital Fernand Widal, Paris, France

Texte reçu le 24 novembre 2014 ; accepté le 5 décembre 2014

\section{Mots clés :}

France ; Europe ;

PCRD ; Horizon 2020 ; information ; éducation ; incitation ; valorisation ; mutualisation
Résumé - Le lancement d'Horizon 2020, 8 è programme cadre de recherche et de développement (PCRD) de l'Union Européenne, doté de 79 milliards d'euros, est l'occasion de faire le bilan de la participation de la France aux précédents PCRD. Elle n'est à la hauteur ni de son poids scientifique, ni de son investissement financier. Alors que la France a contribué au budget recherche de l'Union Européenne ( 7 è PCRD) à hauteur de 16,5 à $17 \%$, le retour monétaire assuré par le financement de projets coordonnés auxquels ses équipes participent est de l'ordre de 12,5 à $13 \%$. C'est un manque à gagner de 600 millions d'euros. Même si la situation doit être nuancée par type d'activité, la France, en recherche clinique, apparaît en retrait et moins engagée dans les appels à projets européens par comparaison avec ses voisins. Si la France possède des atouts (financement assuré de la recherche clinique, structuration de réseaux thématiques, lancement de grands plans nationaux), elle est pénalisée par l'éparpillement des ressources au nom de la politique d'aménagement du territoire, le manque d'approche multidisciplinaire et la méconnaissance du fonctionnement de Horizon 2020 par la communauté médicale et scientifique et les institutions auxquelles elles appartiennent.

Les solutions envisagées pour inciter au dépôt de projets coordonnés ou associant des équipes françaises en recherche clinique et aider à leur montage sont de trois ordres :

- élargir la vision de nos enfants, étudiants et collaborateurs et les aider à s'adapter à la mondialisation des connaissances tout au long de leur cheminement éducatif et professionnel ;

- valoriser les actions européennes pour agir sur le contexte européen et faire évoluer les mentalités ;

- aider et accompagner les porteurs de projets en mutualisant les compétences autour d'un nombre limité de centres d'expertise d'aide au montage.

Abréviations : voir en fin d'article.

\footnotetext{
${ }^{\dagger}$ Les articles, analyses et propositions issus des Ateliers de Giens n'engagent que leurs auteurs et ne préjugent pas de la position de leur organisme de tutelle.
} 


\section{1. État des lieux et contexte}

La table ronde, et c'est sa raison d'être, part du constat de la position modeste, en regard de son potentiel scientifique et médical, occupée par la France dans les initiatives prises par l'Union Européenne en recherche en santé. Frilosité, attentisme, manque de réussite ? L'analyse des explications possibles de cette situation doit conduire à la formulation de recommandations pour la faire évoluer.

Avec un budget de 79 milliards d'euros pour le $8^{\text {è }}$ programmecadre de recherche et de développement européen (PCRD) «Horizon 2020 » qui a démarré en janvier 2014, l'enjeu est de taille, surtout en période de disette financière comme celle que connaissent, en France, les chercheurs et les structures auxquelles ils appartiennent.

\subsection{Les «PCRD», l'instrument majeur d'action en recherche de l'Union Européenne}

Voilà tout juste 30 ans que l'Union Européenne a investi le secteur de la recherche scientifique.

Ces PCRD, financés par l'Union Européenne, correspondent à des dotations distribuées aux acteurs de la recherche Européenne par des appels à projets compétitifs.

Ces programmes-cadres, financés par l'Union Européenne, correspondent à des dotations financières distribuées aux acteurs de la recherche européenne par un mécanisme d'appel à projets.

Aux objectifs initiaux de poursuite du processus d'acquisition des connaissances, de progrès médical et de croissance économique avec la création d'emplois à la clé, s'est ajouté en 2000 au sommet de Lisbonne celui de réduire la «fragmentation des efforts, [l'] isolement [et le] cloisonnement des systèmes nationaux de recherche ». ${ }^{[1]}$

Quadriennaux pour ses 6 premières éditions, la durée des PCRD a été portée à 7 ans à compter du 7 è PCRD (2007-2013). Les budgets consacrés ont été à chaque fois revalorisés : 17,5 Mds d'€ pour le $6^{\text {è }}$ PCRD (2002-2006), 50,5 Mds d'€ pour le 7 è PCRD (2007-2013) et 79 Mds d' $€$ pour Horizon 2020 (le $8^{\text {è }}$ ).

\subsection{Analyse du positionnement de la communauté scientifique française}

Le positionnement et les performances de la communauté scientifique française sont contrastés.

Prise sur un plan global et de manière sans doute un peu simpliste, la situation peut être résumée par la confrontation de deux chiffres avec d'un côté une contribution de la France au budget de l'Union Européenne à hauteur de 16,5 à $17 \%$ et de l'autre un retour monétaire par le financement de projets coordonnés auxquels ses équipes participent de l'ordre de 12,5 à $13 \%{ }^{[2]}$ Sans aller jusqu'à affirmer que la France finance la recherche des Pays-Bas ou d'autres pays plus performants, ces données interpellent. Pour chaque euro investi, la France «n'en récupère » que 70 centimes
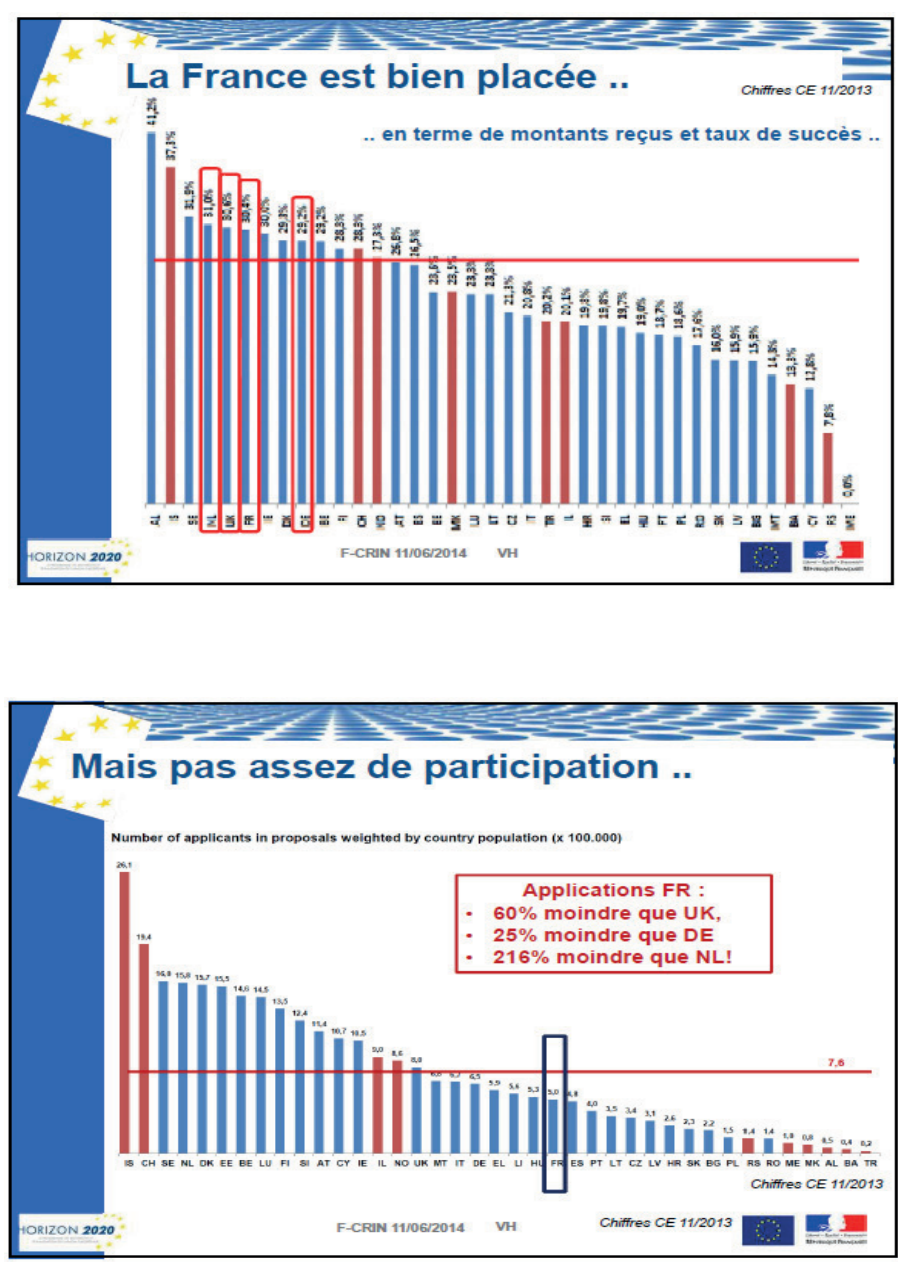

Fig. 1. Comparatif du nombre de projets français et de leur taux de succès dans le FP7 (chiffres Commission Européenne/DG RTD-Novembre 2013) Avec l'aimable autorisation de Guillaume Fusai (Ministère de l'Éducation Nationale, de l'Enseignement Supérieur et de la Recherche).

avec un manque à gagner évalué à 600 millions d'euros, c'est-àdire l'équivalent d'une année de financement de l'Agence Nationale de la Recherche (ANR).

La première question à se poser est bien entendu celle du «pourquoi » de la situation : est-elle liée à une pusillanimité de la communauté scientifique française avec trop peu de projets candidats ou, hypothèse plus dramatique, à des projets de qualité insuffisante ? Il semble, et c'est rassurant, selon les indicateurs disponibles que l'on se place plutôt dans le premier cas que dans le second.

Si l'on s'en tient au taux de succès des projets français, la position occupée au 6 è rang sur 31 Etats-Membres ou partenaires associés est plus qu'honorable, derrière les Pays-Bas et juste derrière la Grande-Bretagne, mais devant l'Allemagne. Lorsque l'on se penche cette fois sur le nombre de projets présentés rapportés à la population, la France est cette fois en bas de liste, au $23^{\mathrm{è}}$ rang (figure 1). 
Un niveau d'analyse supplémentaire en distinguant les performances par grand secteur d'activité et de recherche enrichit la réflexion. Rien de surprenant à ce que les performances françaises soient excellentes en terme de retour sur investissement dans ses secteurs industriels de pointe ( « récupération » de $26 \%$ du total du budget dans le secteur de l'espace; $24 \%$ pour l'aéronautique ; $23 \%$ pour le nucléaire). À l'autre bout de l'échelle, la part du budget « récupéré » est inférieure à $10 \%$ dans le domaine de l'environnement (9\% seulement dans l'action knowledge-based bio-economy), celui des nano-technologies ( $8 \%$ du budget) et $6 \%$ seulement dans les sciences humaines et sociales, là où justement se place une partie peu exploitée en France de la recherche médicale/ clinique.

$\mathrm{Au}$ vu des résultats de la $1^{\text {ère }}$ étape de sélection du « $1^{\mathrm{er}}$ call PHC d'Horizon $2020 »\left(1^{\text {er }}\right.$ semestre 2014), la France reste en retrait si l'on s'en tient au nombre de projets présentés. Elle occupe la $2^{\mathrm{è}}$ place, par contre, après la Finlande, en termes de dossiers présélectionnés avec un score de $50 \%$.

Les résultats finaux de la sélection ne sont pas encore connus (figure 2).

\subsection{Horizon 2020, une chance et des obstacles}

\subsubsection{La recherche clinique, un axe thématique à part entière}

D'abord cantonnés, sur les premières éditions, au seul champ de la recherche fondamentale, les PCRD se sont progressivement ouverts à la recherche appliquée, et donc, dans le domaine de la santé, à la recherche clinique. L'explication de ce recentrage est à rechercher non seulement dans la prise en compte d'une demande exprimée, via les Etats-Membres, d'une partie de la communauté scientifique, mais aussi, dans le souci des instances européennes, de rendre leur politique opérationnelle de soutien et les moyens mis au service de cette politique, plus lisibles et plus concrets pour le citoyen. Ce qu'illustre, sur un plan général, l'introduction dans les dossiers de candidature d'un volet dit « d'impact sociétal ».

L'amorçage s'est opéré à compter du 6 ${ }^{\mathrm{è}}$ PCRD (2003-2007), plus affirmé dans le programme-cadre suivant. Sur plus de 700 projets financés en santé dans les 5 premières années du 7 è PCRD, plus de 300 incluaient des essais cliniques. ${ }^{[3]}$

Axé sur les «défis sociétaux » auxquels la communauté doit faire face, qui constituent l'un de ses trois piliers avec «l'excellence scientifique » et la «primauté industrielle », Horizon 2020 réserve une place de choix à la ligne thématique « Santé ; bien-être et vieillissement». Cette seule thématique regroupe 6,8 Mds d'€, soit $25 \%$ du total, sur les 27 Mds d'€ affectés à l'axe « défis sociétaux».

La valence « recherche clinique » est clairement affirmée dans Horizon 2020 avec des lignes thématiques identifiées et dédiées dans les premiers appels à projets/calls (ligne thématique PHC 152014/15 : «Clinical research on regenerative medicine », ...).

\begin{tabular}{|c|c|c|c|}
\hline Pays & Déposés & Invités & $\%$ \\
\hline FR & 134 & 67 & 50 \\
\hline UK & 208 & 98 & 47 \\
\hline DE & 186 & 83 & 45 \\
\hline NL & 163 & 80 & 49 \\
\hline IT & 224 & 61 & 27 \\
\hline ES & 203 & 60 & 30 \\
\hline AT & 46 & 17 & 37 \\
\hline IE & 41 & 17 & 41 \\
\hline IL & 43 & 15 & 35 \\
\hline BE & 61 & 25 & 41 \\
\hline DK & 46 & 19 & 41 \\
\hline FI & 33 & 17 & 52 \\
\hline SE & 62 & 27 & 44 \\
\hline NO & 33 & 11 & 33 \\
\hline PT & 25 & 5 & 20 \\
\hline SI & 16 & 3 & 19 \\
\hline CH & 17 & 5 & 29 \\
\hline
\end{tabular}

Fig. 2. Appels à projets "Horizon 2020". Résultats de la première étape de sélection des appels à santé "PHC" 2014. http://www.univ-toulouse.fr/sites/ default/files/h2020_sante_20062014.pdf

Avec l'aimable autorisation de Nacer Boubenna, coordinateur H2020, Inserm, Paris (France). Chiffres fournis par la Direction Générale « développement recherche et technologique » de la Commission Europénne.

\subsubsection{Plus de budget, plus d'actions et plus de complexité ?}

En décidant d'agir à l'échelle de l'Europe, l'un des trois grands pôles économiques mondiaux, et en mettant, au service de cette action de soutien de la recherche, des moyens financiers conséquents, les Etats-Membres et les institutions européennes ont fait preuve d'une grande ambition.

Si grande que, pour donner forme à cette force de frappe considérable et faire en sorte qu'elle soit efficace, la mise en œuvre est nécessairement complexe et se décline en «micro-actions » chacune ayant sa cible et sa pertinence. Elles finissent par être si nombreuses que leur multiplicité opacifie la lisibilité par les non-initiés et constitue un frein à l'implication de ceux qui, comme les chercheurs pris dans leur quotidien, n'ont pas nécessairement de temps à consacrer au repérage des initiatives qui pourraient les intéresser.

Les appels à projets ou propositions relevant d'Horizon 2020 peuvent être classés en 3 grandes familles, chacune intégrant une cible différente :

- celles destinées aux chercheurs en tant qu'individus, comme par exemple les «Bourses Marie Curie » qui financent des mobilités pendant un ou deux ans ou encore les European Research Council (ERC) Grants pour des chercheurs éminents qui souhaitent mener une recherche exploratoire ;

- celles destinées à soutenir les partenariats publics/privés tel l'Innovative Medicines Initiative (IMI) qui vise à accélérer le processus de développement de nouveaux produits de santé et leur mise à disposition des patients par un principe de mise en 
commun de moyens, en «cash » pour l'Union Européenne et « in kind » pour les industries de santé et l'Union Européenne sur des thématiques de recherche d'intérêt prioritaire ;

- celles destinées aux équipes de recherche publique (académique, hospitalière et institutionnelle) et accompagnées d'une incitation d'association de partenaires privés (petites et moyennes entreprises/small medium entreprise [PME/SME]), via des appels à projets thématiques tels les personalising health and care (PHC) avec 15 appels en 2014, 17 en 2015.

Bien qu'ayant un impact direct en recherche, d'autres initiatives européennes sortent du champ d'Horizon 2020 :

- soit parce qu'elles ont un champ plus vaste que la seule activité de recherche \& développement (R\&D), comme les fonds structurels européens qui visent à renforcer la solidarité entre les Etats-Membres en réduisant les inégalités de développement. On en retiendra trois types : Fonds Européen de Développement Régional (FEDER) ; Fonds Social Européen (FSE) et le fonds « de cohésion ». Ces fonds font l'objet de négociations directes entre l'Union Européenne et chaque EtatMembre (qui, comme c'est le cas pour la France, associent en général les collectivités régionales);

- soit parce qu'ils relèvent d'autres sources de financement nationaux coordonnés à l'échelle européenne, tels les initiatives de programmation conjointe (Joint Programming Initiatives [JPIs]) ou les European Research Area Network (ERANETs) dont l'objectif est de mutualiser et coordonner, au niveau européen, des initiatives nationales comparables sur des mêmes thématiques. Là, le financement des projets de recherche est apporté par les Etats-Membres via les agences de financement participant complété par un financement de l'UE pour les activités de coordination ou des « top up » des financements nationaux pour certains appels à projets (cofinancement de programmes régionaux, nationaux et internationaux [CO-FUND]).

Difficile, dans ce maquis touffu de mécanismes divers et variés, de prétendre tous les analyser. Poussés par la nécessité et l'impératif d'efficacité, les participants à la table ronde ont décidé de focaliser leur attention sur les deux instruments les plus connus des institutions et organisations œuvrant dans le domaine de la recherche clinique, à savoir les PHC et le partenariat public/privé « IMI».

\section{Analyse des points positifs et négatifs de l'environnement et de l'organisation Française}

L'objectif de notre table ronde étant de déterminer des axes stratégiques pour la France qui permettraient d'atteindre un objectif concret d'augmentation des taux de participation et de succès des équipes françaises dans le cadre des appels à projets européens Horizon 2020, nous avons choisi d'appliquer la méthodologie dite du strengths-weaknesses-opportunities-threats (SWOT).

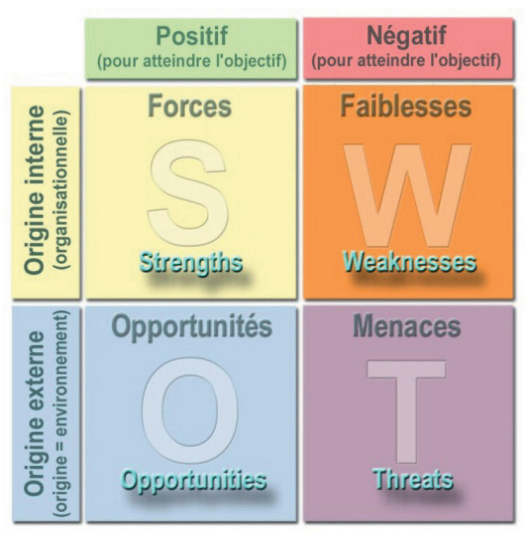

Fig. 3. La représentation du SWOT se fait sous forme de matrice.

\section{1. La méthodologie du SWOT}

Définition : l'analyse (ou la matrice) SWOT est définie par les services de la Commission Européenne comme « un outil d'analyse stratégique qui combine l'étude des forces et des faiblesses d'une organisation, d'un territoire, d'un secteur... avec celle des opportunités et des menaces de son environnement, afin d'aider à la définition d'une stratégie de développement. »

À ce titre, l'analyse (ou la matrice) SWOT fait partie des outils qui aident à la mise en place de stratégie futures à mettre en œuvre en prenant en compte l'environnement actuel et futur (qu'il s'agisse d'une action ponctuelle ou d'une stratégie d'ensemble).

Le terme SWOT est un acronyme issu de l'anglais (figure 3) :

- Strengths (forces) ;

- Weaknesses (faiblesses) ;

- Opportunities (opportunités) ;

- Threats (menaces).

\subsection{Conduite et construction du SWOT au cours de la table ronde}

Les sujets discutés ont été orientés sur l'organisation, les compétences et les systèmes français et non sur des thématiques scientifiques. Cette analyse a été conduite avec l'ensemble des participants à la table ronde (académiques, institutionnels et personnels de l'industrie pharmaceutique), et enrichie par les interviews menées au préalable (institutions et biotechs).

\subsection{SWOT de la situation actuelle de la France dans le cadre des appels à projets européens}

La situtation de la France est représentée dans la figure 4. 


\begin{tabular}{|c|c|}
\hline $\begin{array}{l}\text { FORCES } \\
\text { - Expertise scientifique, médicale, esprit de créativité et d'innova- } \\
\text { tion reconnus } \\
\text { - Existence, contrairement à beaucoup d'autres pays, de systèmes } \\
\text { de soutien financier à la recherche clinique (ex : PHRC) } \\
\text { - Investissements dans des « Pôles » d'excellence (ex : Instituts } \\
\text { (IHU) et Départements Hospitalo-Universitaires [DHU]) } \\
\text { - Réseaux thématiques structurés et labellisés } \\
\text { - Compétences managériales de haut niveau (ingénieurs issus des } \\
\text { grandes écoles) } \\
\text { - Existence de structure d'aide au montage \& au suivi de projets } \\
\text { européens (ex : Inserm-Transfert, ECRIN) } \\
\text { - "Success stories » françaises reconnues }\end{array}$ & $\begin{array}{l}\text { FAIBLESSES } \\
\text { - Faiblesse de l'analyse de l'impact sociétal } \\
\text { - Maîtrise de l'anglais et ouverture à l'international insuffisant } \\
\text { - Complexité du système français : « trop de guichets tue le guichet »... } \\
\text { - Une politique d'aménagement du territoire non ciblée sur la localisation réelle } \\
\text { des compétences } \\
\text { - Multiplicité des sources nationales de financement pouvant freiner l'engage- } \\
\text { ment européen } \\
\text { - Silotage des éducations : «il y a de la compétence mais on ne se parle pas » } \\
\text { - Problématiques «RH » dans les structures publiques : attractivité statutaire et } \\
\text { salariale faible : perte de talents, non attractivité des personnels hautement } \\
\text { qualifiés } \\
\text { - Culture partenariale «public/privé » insuffisante } \\
\text { - Impact trop faible du lobbying français auprès des institutions européennes } \\
\text { - Absence de reconnaissance académique de l'engagement dans des projets } \\
\text { européens } \\
\text { - Méconnaissance et perception négative par les potentiels coordinateurs / } \\
\text { investigateurs }\end{array}$ \\
\hline $\begin{array}{l}\text { OPPORTUNITÉS } \\
\text { - Évolution de la réglementation européenne : «Medical device » } \\
\text { \& «Clinical trials } \\
\text { - Nouvelles opportunités de financement européens (Eranet, Joint } \\
\text { programming initiatives [JPIs]) } \\
\text { - Dynamique créée par ECRIN et les investissements d'avenir : } \\
\text { F-CRIN, IHU (et ce qui en découle = DHU) } \\
\text { - Mise en œuvre de réseaux thématiques multinationaux d'investi- } \\
\text { - Concrétisation des actions du CSIS }\end{array}$ & $\begin{array}{l}\text { MENACES } \\
\text { - «Le temps est compté » : nos voisins européens sont de plus en plus perfor- } \\
\text { mants. La France montre des difficultés à définir une stratégie sur les axes } \\
\text { d'excellence (exemple de nos voisins : Grande Bretagne = choix de } \\
7 \text { domaines prioritaires) } \\
\text { - Risque du recours exclusif aux structures privées pour montage et suivi projet } \\
=\text { externalisation de la compétence (« on apprend peu et ça coûte cher ») } \\
\text { - Risque de relégation des équipes françaises au rôle d'effecteur et de contrib- } \\
\text { uteur avec perte de leadership } \\
\text { - Les bons centres français risquent d'être «noyés » dans des consortiums coordon- } \\
\text { nés par des centres étrangers = manque de visibilité et risque de fuite des experts }\end{array}$ \\
\hline
\end{tabular}

CSIS : Center for Strategic and International Studies ; DHU : Département Hospitalo-Universitaire ; ECRIN : European Clinical Research Infrastructures Network; F-CRIN : French Clinical Research Infrastructure

Network; IHU : Institut Hospitalo-Universitaire ; Inserm : Institut National de la Santé et de la Recherche Médicale ; PHRC : programme hospitalier de recherche clinique ; RH : ressources humaines ; SWOT : strengths-weakness-opportunities-threats (forces-faiblesses, opportunités, menaces).

Fig. 4. Analyse SWOT du positionnement de la France dans les projets européens.

Nous souhaitons développer certains points :

- concernant les forces, nous soulignons que l'une des forces de la France est l'ensemble des systèmes, efficaces et rodés de financement de la recherche clinique. Ces subventions ont permis aux équipes françaises de développer des projets, de confirmer et renforcer leur excellence scientifique. Ces projets nationaux sont considérés également comme de bons tremplins pour permettre dans un second temps un engagement dans des projets européens (figure 5);

- soulignons cependant que ces financements peuvent devenir aussi une faiblesse, la connaissance et l'habitude de présenter des projets nationaux devenant potentiellement un frein à l'engagement dans des projets européens. Concernant les
Biotechs, les différentes possibilités de financement national ne privilégient pas les projets européens, lesquels sont plus longs à mettre en œuvre et consommateurs de ressources, critères parfois difficilement compatibles avec la structure de ces PME et leur échelle de temps ;

- nous notons également que la structuration des réseaux thématiques et le lancement de grands plans nationaux (plan maladies rares », plan «cancer», plan Alzheimer...) créent des dynamiques profitables à l'engagement dans les projets européens ;

- enfin, si l'on s'intéresse aux compétences managériales de conduite de projet et d'analyse d'impact sociétal, la France est dotée d'écoles de haut niveau, reconnues internationalement. 


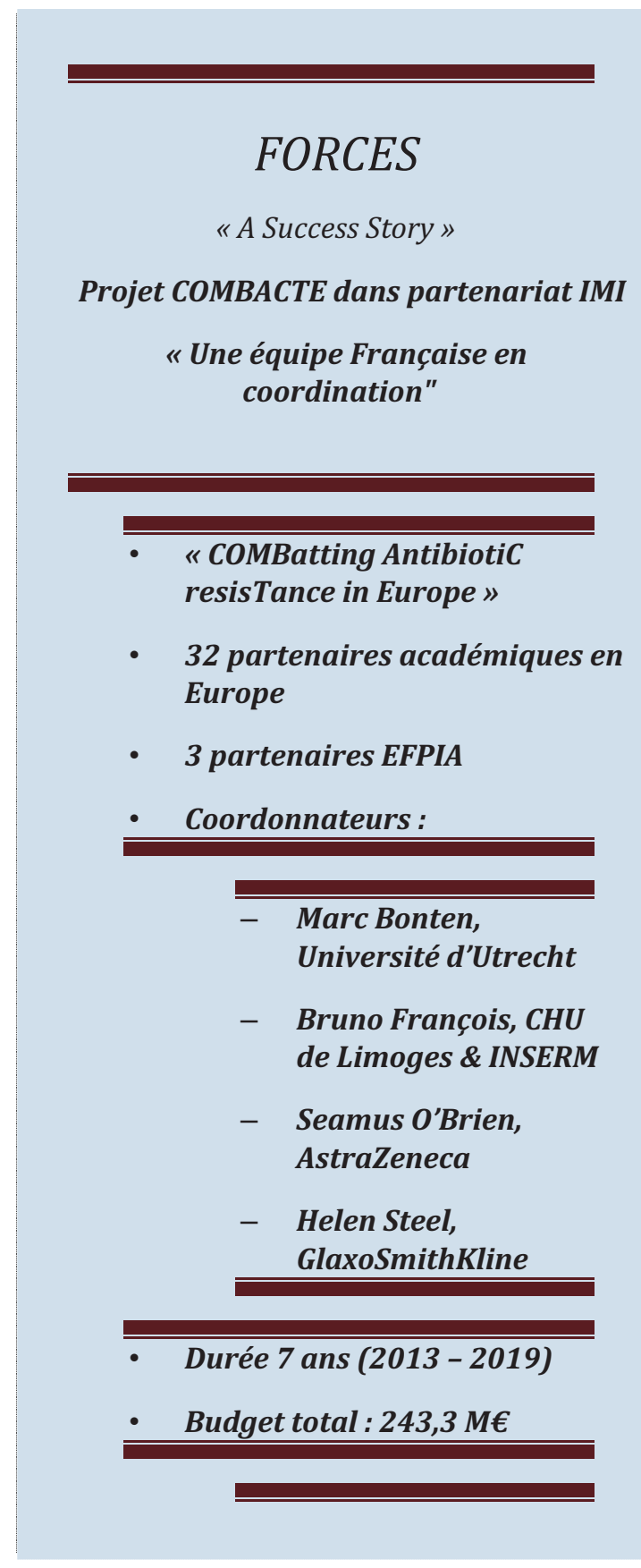

Fig. 5. Présentation du projet IMI "COMBACTE" (COMBatting AntibiotiC resisTance in Europe).

CHU : Centre Hospitalo-Universitaire ; EFPIA : Fédération Européenne des Industries et Associations Pharmaceutiques; IMI: Innovative Medicines Initiatives ; Inserm : Institut National de la Santé et de la Recherche Médicale.

L'expertise est bien réelle dans notre pays, mais le manque d'approche multidisciplinaire ne permet pas de mettre à profit la synergie des compétences indispensable dans ce type de projets.
Concernant les faiblesses, la politique d'aménagement du territoire de la France conduit à un éparpillement des ressources sans tenir compte de la localisation réelle des compétences.

Un autre point trivial, mais essentiel, est la méconnaissance du fonctionnement d'Horizon 2020 par certains acteurs qui introduit une perception négative et un frein ici encore à l'engagement. La labellisation des réseaux (French Clinical Research Infrastructure Network [F-CRIN]) et le déploiement des investissements d'avenir offrent une aide très efficace à la collaboration nationale et internationale.

Enfin, il est reconnu qu'il est urgent que la France réagisse et contribue à des changements de mentalités et d'approche, car nos pays européens voisins sont de plus en plus performants et le retard sera de plus en plus grand et difficile à rattraper.

Les résultats de cette première analyse nous ont permis de proposer une liste de recommandations pour soutenir une stratégie française efficace et gagnante.

\section{Les recommandations}

Les faiblesses de la communauté française vis-à-vis de sa capacité à répondre aux appels à projets européens par rapport aux autres pays, en particulier concernant le domaine de la biologie et de la santé, sont en rapport en premier lieu avec un enfermement linguistique. Ce fait est à ce point ancré dans notre système d'éducation et de professionnalisation qu'elle a conduit les membres de cette table ronde à éditer des recommandations dont certaines concernent le début du cursus éducatif.

Nous les classerons en trois grands axes.

Élargir la vision de nos enfants, étudiants et collaborateurs et les aider à s'adapter à la mondialisation des connaissances tout au long de leur cheminement éducatif et professionnel

1. Accentuer l'apprentissage des langues et des civilisations étrangères en multipliant les expériences dans d'autres pays pour nos lycéens, sous forme de séjours dit « linguistiques »- mais qui sont largement plus formateurs que le simple langage. Ces expériences ne doivent naturellement pas se limiter à l'Europe. En Faculté de Médecine, un certain nombre de cours (pour la lecture critique d'articles par exemple) pourraient être donnés en langue anglaise. Le Ministère de l'Éducation doit jouer un rôle incitatif majeur.

2. Rendre obligatoire la mobilité des étudiants effectuant des études en biologie et en santé au cours de leur cursus, à des moments bien choisis afin que cette mobilité soit vécue comme attractive et non source potentielle d'échec aux contrôles théoriques. Privilégier les stages qui mettent les étudiants en contact avec des équipes étrangères qui ont déjà des liens avec leur université. Ceci 
permet à l'étudiant de comprendre comment les chercheurs travaillent en réseau et comment s'y intégrer, et de programmer un autre stage à un point différent de ce réseau. Les trois conférences des doyens en santé (médecine ; pharmacie ; odontologie) doivent être les maîtres du jeu.

3. Orchestrer la montée en charge des relations entre médecins, autres professionnels de santé, enseignants et chercheurs de différents pays, en particulier européens. Cette politique hospitalo-universitaire internationale devrait être au mieux effectuée en concertation entre les universités et l'Alliance Nationale pour les Sciences de la Vie et de la Santé (AVIESAN). Rendre obligatoire une mobilité réelle et prolongée pour les promotions académiques comportant un ou plusieurs séjours à l'étranger de type $P h D, v i$ siting professor...

Valoriser l'accueil de collègues étrangers pour des séjours de plusieurs mois et les échanges de personnel au sein d'un réseau international qui, actif et dynamique, deviendra le berceau de projets européens ou mondiaux. Ce sont ces éléments issus de cette politique concertée qui devraient influencer les indicateurs des jurys d'évaluation et de promotion des personnels, en concertation avec le Ministère de l'Enseignement Supérieur et de la Recherche (MESR).

Valoriser les actions européennes pour agir sur le contexte européen et faire évoluer les mentalités

4. Rendre les appels à projets nationaux plus proches de la structure des appels européens : rédaction en anglais pour tous (y compris le PHRC régional), augmentation de la part de l'impact du projet, en particulier du point de vue socio-économique... Ces actions concernent le Ministère de la Santé, le MESR et l'ANR.

5. Augmenter la visibilité européenne du tissu des PME françaises afin que les porteurs de projet académiques français et étrangers puissent les contacter facilement et inversement que les PME françaises puissent trouver rapidement les partenaires académiques dont ils ont besoin. Ce rôle pourrait être dévolu aux pôles de compétitivité et au Ministère de l'Industrie.

6. Multiplier les actions d'information/formation régionales ciblées sur les équipes potentiellement aptes de générer et mener à bien un projet européen au sein d'un réseau déjà actif. Ces actions pourraient être renforcées et mieux coordonnées en unissant les forces des PCN, de F-CRIN, des universités, des Établissements Publics à Caractère Scientifique et Technologique (EPST) et du Comité National de Coordination de la Recherche (CNCR).

7. Motiver les experts français à s'intégrer dans une démarche européenne :

- en devenant évaluateurs de projets européens - ce qui est très formateur pour conduire de futurs projets - en incluant cet item dans la politique hospitalo-universitaire internationale déjà citée qui devrait inciter les jurys d'évaluation et de promotion des personnels à insérer ce critère dans leur grille ;
- en prenant la responsabilité d'un work package dans un projet européen, une première étape pour comprendre la mécanique administrative et se porter coordinateur d'un projet européen.

8. Valoriser le dépôt et la réussite aux appels à projets européens auprès des jurys d'évaluation et de promotion des personnels et des structures dans la même veine que précédemment.

9. Définir des domaines prioritaires pour la France, sources privilégiées d'investissements qui amélioreraient encore la crédibilité de la politique hospitalo-universitaire internationale. L'ensemble des trois Ministères (Santé, MESR, et Industrie) ainsi qu'AVIESAN devraient être parties prenantes.

10. Renforcer et coordonner le lobbying français à Bruxelles, en tenant compte de ces domaines prioritaires avec l'aide conjointe des PCN, des universités, du CNCR, des EPST et du MESR.

\section{Aider les porteurs de projets}

11. Concentrer les compétences qui sont rares, en les mutualisant autour d'un nombre limité de centres d'expertise d'aide au montage et au suivi de projets européens en recherche clinique, correctement financés, ceci afin d'éviter le saupoudrage sur de multiples mini-structures inefficaces. Seraient impliqués dans cette concertation, AVIESAN, le CNCR, les universités.

12. Assurer le caractère transdisciplinaire du projet, en insistant sur :

- l'expertise méthodologique spécifique aux grandes cohortes de patients ;

- l'expertise informatique adaptée à la gestion d'un nombre très important de données de multiples sources ;

- l'expertise économique et sociétale ;

- et enfin l'expertise de transfert de technologie qui font souvent la différence au moment de la sélection finale. Les universités, AVIESAN, l'Alliance pour la Recherche et l'Innovation des Industries de Santé (ARIIS) et les « big pharmas » devraient y jouer un rôle majeur.

13. Faire comprendre aux porteurs de projet l'importance d'un ingénieur en management pour la mise en œuvre et le suivi de leur projet. Les écoles d'ingénieurs, les grandes écoles et les structures d'expertise et de soutien existantes (exemple : certaines cellules Europe des Centres Hospitalo-Universitaires [CHU]) pourraient apporter une contribution essentielle.

14. Donner aux porteurs de projets les moyens, la responsabilité et l'autonomie d'une gestion financière, ainsi que des ressources humaines professionnelles au sein des structures de montage et de suivi déjà évoquées.

15. Enfin, mettre en place les ressources et l'expertise nécessaires au sein d'un nombre très limité de structures de montage spécialisées à l'évaluation des dispositifs médicaux. Les institutions impliquées seraient les universités, les écoles d'ingénieurs, les pôles de compétitivité, le Ministère de la Santé, de l'Industrie et de l'ESR, F-CRIN. 


\section{Conclusion}

Quinze propositions ont été identifiées regroupées en trois axes d'actions :

- élargir la vision de nos enfants, étudiants et collaborateurs et les aider à s'adapter à la mondialisation des connaissances tout au long de leur cheminement éducatif et professionnel ;

- valoriser les actions européennes pour agir sur le contexte européen et faire évoluer les mentalités ;

- aider et accompagner les porteurs de projets en mutualisant les compétences autour d'un nombre limité de centres d'expertise d'aide au montage.

Ces recommandations doivent maintenant être portées au plus haut niveau des pouvoirs publics pour devenir réalité. Elles ne sont pas coûteuses et relèvent souvent du bon sens et de la réorganisation, de la réallocation ou de l'accélération d'évolutions déjà engagées.

Les instances représentant la recherche clinique et fédérant ses acteurs, AVIESAN pour la recherche académique, ARIIS pour les industriels de santé et le CNCR pour la communauté hospitalière, pourraient être les porteurs de ce plan d'action.

Conflits d'intérêts. Les auteurs n'ont pas déclaré de conflits d'intérêts en lien avec cet article.

Abréviations. ANR: Agence Nationale de la Recherche; ARIIS : Alliance pour la Recherche et l'Innovation des Industries de Santé ; AVIESAN : Alliance Nationale pour les Sciences de la Vie et de la Santé; CHU: Centre Hospitalo-Universitaire; CNCR : Comité National de Coordination de la Recherche ; COFUND : cofinancement de programmes régionaux, nationaux et internationaux ; EPST : Établissement Public à Caractère Scientifique et Technologique; ERA-NETs : European Research Area Network; ERC : European Research Council ; FEDER : Fonds Européen de Développement Régional; FSE: Fonds Social Européen ; IMI : Innovative Medicines Initiative ; JPIs : initiatives de programmation conjointe (Joint Programming Initiatives) ; MESR: Ministère de l'Enseignement Supérieur et de la Recherche; PCRD: programme cadre de recherche et de développement; $\mathrm{PHC}$ : personalising health and care; PME : petites et moyennes entreprises ; R\&D : recherche \& développement; SWOT : strenghts-weakness-opportunities-threats.

\section{Références}

1. Busquin P. Membre de la Commission Européenne en charge de la recherche. L'impact des technologies de l'information et de communication sur l'Espace européen de la recherche. Amsterdam, 7 décembre 2000 http://europa.eu/rapid/press-release_SPEECH-00-496_fr.htm Consulté le 5 décembre 2014

2. http://www.performance-publique.budget.gouv.fr/ Consulté le 5 décembre 2014

3. http://www.europa.eu/ Consulté le 5 décembre 2014

Correspondance et offprints : Jean-François Dhainault, Président du GIRCI "Ile de France », Carré historique, Secteur gris, Porte 23, Hôpital Saint Louis, 1 avenue Claude Vellefaux, 75010 Paris, France

E-mail : jean-francois.dhainaut@drc.aphp.fr

Vincent Diebolt, F-CRIN, Immeuble AROPA, 35 rue Bernard de Ventadour, 31300 Toulouse, France

E-mail : vincent.diebolt@inserm.fr

Brigitte Pouletty-Lefebvre, Laboratoire Sanofi France, 9 boulevard Romain Rolland, 75159 Paris cedex 14, France.

E-mail : brigitte.pouletty-lefebvre@sanofi.com 